\section{Tecnologia para acesso à água na várzea amazônica: impactos positivos na vida de comunidades ribeirinhas do Médio Solimões, Amazonas, Brasil}

Technology for access to water in the Amazonian floodplain: positive impacts on the lives of riverine communities in the Middle Solimões, Amazonas State, Brazil

Tecnología para acceso al agua en la cuenca amazónica: impactos positivos en la vida de comunidades ribereñas del Médio Solimões, Amazonas, Brasil

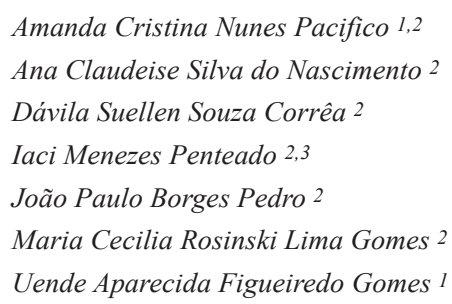

doi: 10.1590/0102-311X00084520

\section{Resumo}

Este estudo apresenta uma avaliação dos impactos de saúde e social com base na implantação de sistemas de abastecimento de água em comunidades ribeirinhas na Região do Médio Solimões, Estado do Amazonas, Brasil. Os dados foram levantados em 21 comunidades rurais, dentre elas 19 que receberam a tecnologia de acesso à água de forma experimental nos últimos 14 anos. Para a comparação, participaram da pesquisa três comunidades rurais que não receberam a tecnologia. A metodologia utilizada foi quanti/quali, com aplicação das técnicas: grupos focais, entrevistas semiestruturadas, questionários, exames parasitólogicos e análise da qualidade da água. Avaliou-se que mesmo não ocorrendo a redução da prevalência de parasitoses, os sistemas apresentaram impactos quanto ao conforto, a privacidade e na redução do tempo gasto em atividades domésticas, como buscar água no rio ou lavar roupas e vasilhas fora do domicílio. Os impactos apontam para a melhoria das condições de vida com base na perspectiva do bem-estar.

Abastecimento de Água; Ecossistema Amazônico; Desenvolvimento Sustentável; Água
Correspondência

A. C. S. Nascimento

Instituto de Desenvolvimento Sustentável Mamirauá. Rua Espírito Santo 1146, Tefé, AM 69553-340, Brasil. claudeise@mamiraua.org.br

1 Universidade Federal de Minas Gerais, Belo Horizonte, Brasil. 2 Instituto de Desenvolvimento Sustentável Mamirauá, Tefé, Brasil.

3 Conservação Internacional, Rio de Janeiro, Brasil. 


\section{Introdução}

O abastecimento de água é componente do saneamento básico, conforme estabelecido pela Lei no 11.445/2007 1. Apesar de reconhecido como um direito humano fundamental pela Organização das Nações Unidas (ONU), a universalização do acesso à água constitui um grande desafio político, social e tecnológico.

O Estado do Amazonas apresenta um dos piores indicadores de abastecimento de água do Brasil. Dados do Instituto Brasileiro de Geografia e Estatística (IBGE) apontam que 60\% da população rural do Amazonas retiram a água para o consumo humano diretamente dos rios, igarapés, lagos ou açudes 2. Nesse estado, o acesso à cobertura por rede geral de abastecimento de água somente alcança $10 \%$ da população 2 . Esse panorama evidencia que o acesso à água potável em áreas rurais brasileiras ainda hoje é incipiente, agravado diante da ausência de uma política nacional de saneamento rural ${ }^{3}$.

No Brasil, as ações de saneamento são desiguais em razão de seu diverso e extenso território, de suas características físicas e culturais, bem como da omissão do Estado. Diante da ausência de intervenção estatal nas comunidades ribeirinhas da Região Norte, projetos pioneiros são desenvolvidos por instituições da sociedade civil que buscam promover o acesso ao saneamento básico. Entre esses projetos destacam-se as iniciativas: Sistemas de Abastecimento de Água do Projeto Saúde e Alegria (Pará), Sanear do Memorial Chico Mendes (Amazonas) e Água em Casa, Limpa e Saudável da Cáritas Brasileira (Pará). Esses projetos buscam possibilitar o abastecimento de água potável para comunidades que sofrem com a escassez de água de qualidade 4,5,6.

Neste artigo é analisado o modelo do Sistema de Abastecimento de Água (SAA) desenhado e reaplicado pelo Instituto de Desenvolvimento Sustentável Mamirauá (IDSM) para comunidades ribeirinhas da região do Médio Rio Solimões, no Estado do Amazonas. Essas são sazonalmente alagadas por um período de até seis meses e nos outros meses passam por um longo período de escassez de água 7.

Em 2000, foi instalado o primeiro protótipo do SAA, sendo que, até 2014, foram implantados 21 sistemas em comunidades localizadas em duas unidades de conservação. A iniciativa foi motivada pelo interesse em reduzir a prevalência de diarreia nesses locais, considerando os diversos estudos que comprovam a melhoria da saúde em função dos investimentos em abastecimento de água 8 . Essas experiências permitiram aprimorar esse modelo para comunidades da várzea amazônica tanto em aspectos técnicos quanto na sua gestão comunitária ${ }^{9}$.

Avaliar projetos como esses, pensados para realidades heterogêneas em que os serviços básicos de saneamento são precários ou inexistentes, torna-se fundamental para o debate das políticas públicas. Considerando a ausência de estudos de impacto de tecnologias implementadas para suprir as necessidades básicas da população rural, tal qual a demanda por saneamento básico no contexto dos ambientes alagáveis, este artigo apresenta uma avaliação de impactos socioambientais nas comunidades onde os SAAs foram implantados, com enfoque nos aspectos sociais e de saúde.

\section{Avaliação de impactos}

A metodologia de avaliação 10 supõe a existência de relações causais; a avaliação busca responder se o projeto funcionou ou não e descobrir as causas para tal. Em uma avaliação ex-post - após o término da intervenção - tenta-se estabelecer o grau de eficácia e determinar se o projeto atingiu os objetivos programados. As avaliações de impacto, quando realizadas apenas em laboratórios, são curtas, o que impede a avaliação, por um lado, da sustentabilidade a longo prazo e, por outro, do desempenho da tecnologia com base na participação qualitativa dos usuários 11 .

A pesquisa avaliativa verifica se os objetivos previamente estabelecidos foram alcançados e abre espaço para considerar efeitos não previstos, positivos ou negativos, possibilitando assim refletir sobre êxito ou fracasso da iniciativa. Para que isso ocorra, a avaliação de impactos de programas ou projetos deve ir além de aspectos quantitativos como o número de comunidades atendidas, a renda gerada ou a redução das emissões de carbono, mas também devem ser considerados os impactos qualitativos como a transformação das relações sociais e da rotina dos moradores a partir de uma intervenção, aspectos que escapam aos indicadores mais convencionais 10. Pesquisas realizadas na Etiópia 12, 
Gana, Índia e Tanzânia 13 demonstram não só os impactos quantitativos de sustentabilidade dos projetos, mas também os impactos na saúde física e mental, na educação, na vida sociocultural e na renda das comunidades.

\section{Características da tecnologia}

OSAA funciona por meio de energia solar fotovoltaica e capta água da superfície dos rios, bombeando-a até um reservatório central (Figura 1). Nesse reservatório, é realizado seu pré-tratamento (remoção de sólidos grosseiros) e distribuição por canalização para os domicílios. Essa solução tecnológica foi ajustada à realidade socioambiental da várzea amazônica e por isto utiliza estruturas móveis, recurso madeireiro local, energia solar e força de trabalho dos usuários 9.

O SAA não tem capacidade técnica para tornar a água potável, pois o filtro usado não garante o padrão nacional de potabilidade do Ministério da Saúde 1. Todavia, ações complementares, como orientações aos usuários sobre técnicas de tratamento domiciliar simplificado de água, foram realizadas buscando melhorar a qualidade da água do SAA. Uma redução de $20 \%$ na morbidade por diarreia é esperada a partir do acesso à maior quantidade de água 14 . Estudo 15 demonstra que pequenas orientações, como filtrar e ferver a água, podem ter grande impacto na redução da mortalidade infantil, principalmente em zonas rurais ou áreas ocupadas por populações de baixa renda, que não têm acesso aos serviços de fornecimento de água tratada para o consumo humano.

\section{Áreas de estudo e comunidades estudadas}

A área de estudo compreende as comunidades ribeirinhas (Figura 2) localizadas na Reserva de Desenvolvimento Sustentável Mamirauá (RDSM), criada em 1996, com extensão territorial de 1.124.000ha, e a Reserva de Desenvolvimento Sustentável Amanã (RDSA), criada em 1998, com 2.350.000ha, ambas geridas pelo governo do Estado do Amazonas. Abrigam em seus limites aproximadamente 16 mil pessoas em mais de 3 mil domicílios, distribuídos em 337 pequenos agrupamentos populacionais, identificados por comunidades, com uma média de 10 domicílios cada 16.

Tanto a RDSM quanto a RDSA integram o desenho territorial das Unidades de Conservação da Amazônia Central, reconhecidas como Reserva da Biosfera pela Organização das Nações Unidas para a Educação, a Ciência e a Cultura (UNESCO). Dada a sua importância ecológica e na condição de Patrimônio Natural da Humanidade, tem recebido um conjunto de projetos experimentais e uma diversificada rede de intervenções sociais por parte de órgãos governamentais e não governamentais desde a década de 1990.

Na várzea amazônica, o nível de água dos rios dá o ritmo à vida e os moradores estão sujeitos tanto às alagações anuais, que cobrem as terras, as casas e as plantações, quanto às secas, que dificultam a locomoção e o acesso à água para consumo 17.

Essa sazonalidade hídrica leva a uma grande variação na distância entre o domicílio e a margem do rio, podendo chegar a $1 \mathrm{~km} 18$ na época da seca nas comunidades estudadas, com uma amplitude média anual do nível de água de 10,6m 7. Essa variação implica dificuldades para as comunidades ribeirinhas, cujos moradores precisam enfrentar longas distâncias e íngremes barrancos para abastecer seus domicílios com água para beber e cozinhar.

As moradias nessas comunidades são usualmente construídas de frente para o rio, acompanhando o seu curso. Cada domicílio costuma ter um pequeno "porto" - uma balsa com ou sem cobertura - localizada no rio em frente à sua moradia. Como a grande maioria dessa população não possui sistemas de abastecimento de água com distribuição domiciliar 17,19, além de servirem para atracar embarcações, essas balsas são usadas para atividades diárias como tomar banho e lavar roupas e utensílios domésticos.

A higiene pessoal e alimentar é um desafio, uma vez que sem água no domicílio não é possível investir em melhorias como um sistema sanitário de descarga de excretas ou uma pia com sabão para lavar as mãos. A disposição de fezes e urina tende a ser feita de forma precária, em fossas rudimentares 


\section{Figura 1}

Sistema de Abastecimento de Água do Instituto de Desenvolvimento Sustentável Mamirauá (IDSM).

1a) Reservatório de água na época da seca

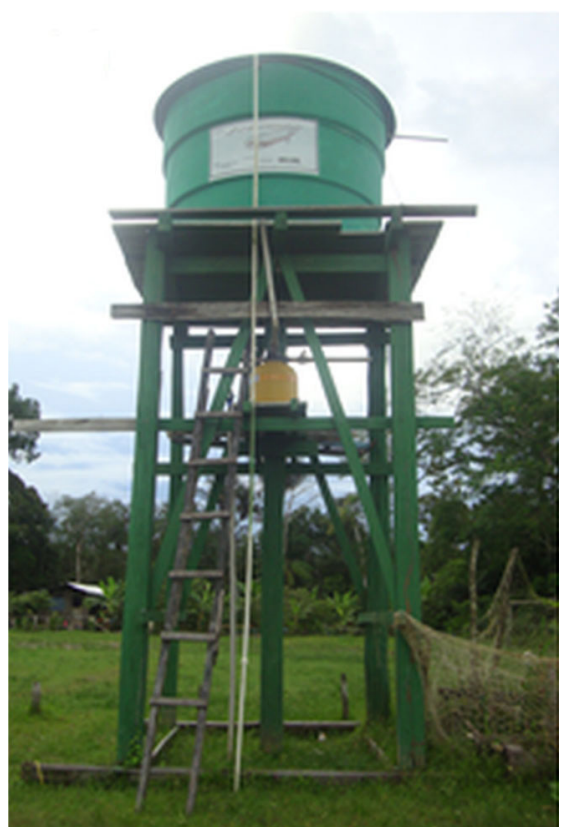

1b) Reservatório de água na época da cheia

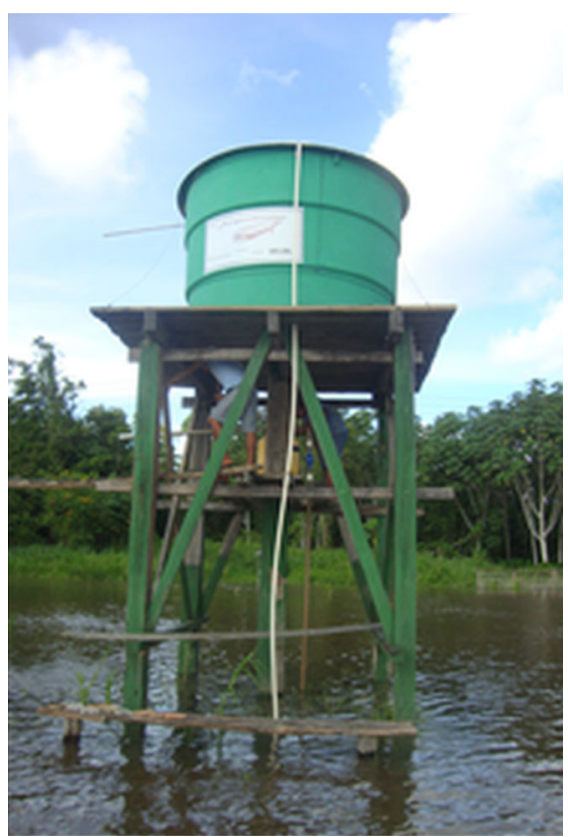

1c) Balsa flutuante com os módulos solares e a bomba d'agua

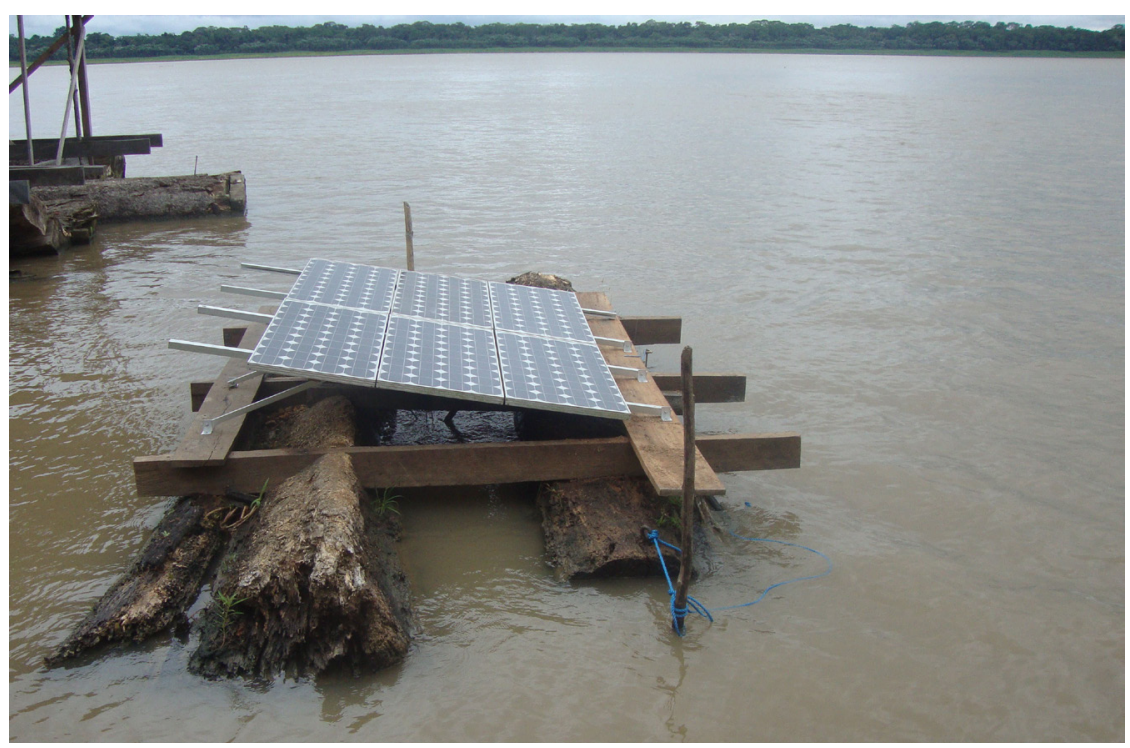

Fonte: Arquivos do Instituto de Desenvolvimento Sustentável Mamirauá (IDSM). 


\section{Figura 2}

Mapa das comunidades ribeirinhas inseridas nas Reservas de Desenvolvimento Sustentável (RDS) Mamirauá e Amanã, Amazonas, Brasil.

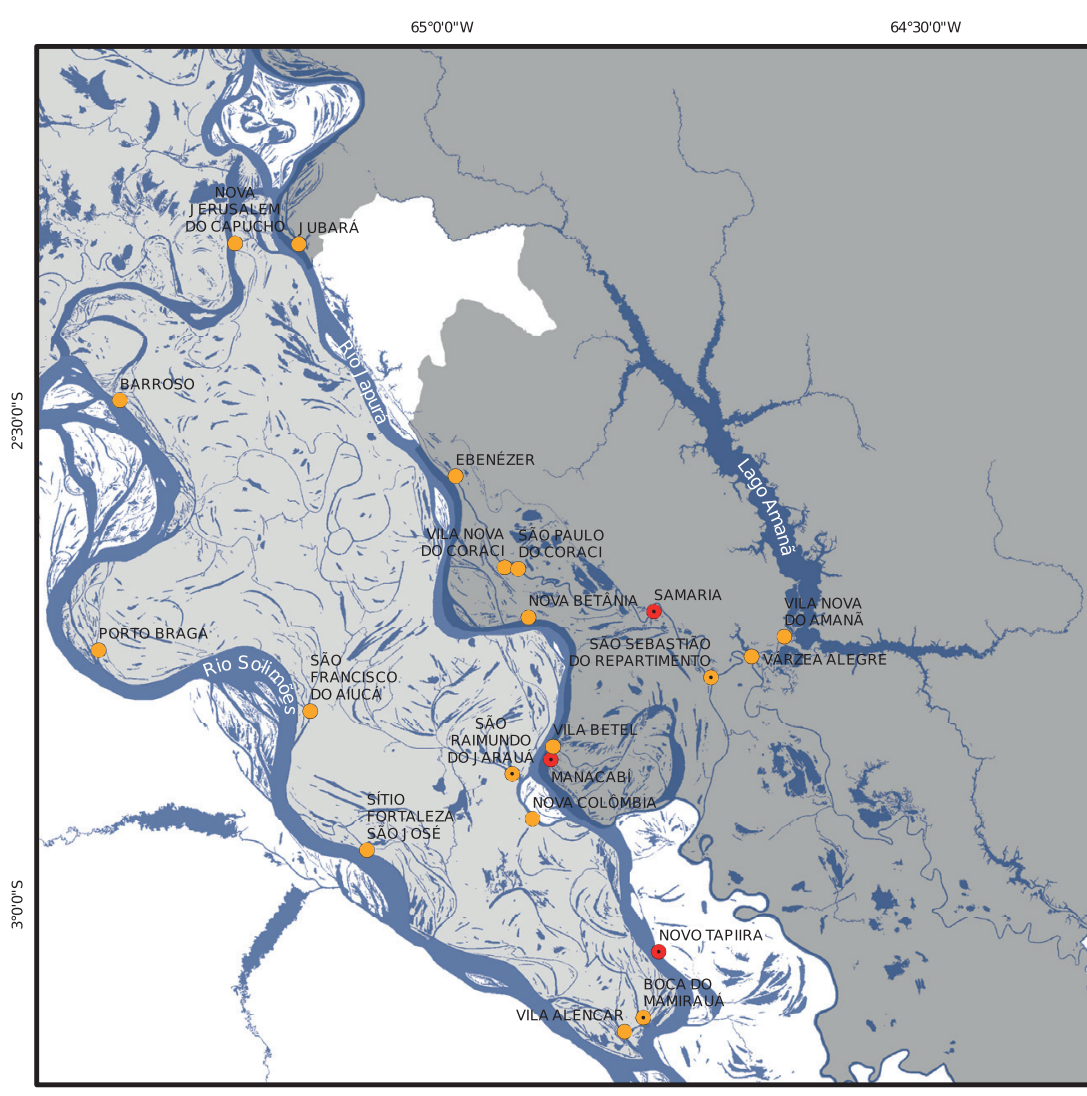

\begin{tabular}{|c|c|c|c|}
\hline \multicolumn{4}{|c|}{ RDS Amanã } \\
\hline \multicolumn{4}{|c|}{ RDS Mamirauá } \\
\hline & alidades & opulação & Domicílios \\
\hline & Manacabí & 123 & 17 \\
\hline & Novo Tapiira & 56 & 13 \\
\hline & Samaria & 45 & 9 \\
\hline$\odot$ & Bocado Mamirauá & 66 & 15 \\
\hline$\odot$ & São S. do Repartimento & 65 & 9 \\
\hline$\odot$ & São Raimundo do Jarauá & 163 & 35 \\
\hline & Barroso & 76 & 14 \\
\hline & Ebenézer & 83 & 14 \\
\hline & Jubará & 124 & 22 \\
\hline & Nova Betânia & 86 & 14 \\
\hline & Nova Colômbia & 44 & 8 \\
\hline & Nova Jerusalém & 56 & 12 \\
\hline & Porto Braga & 161 & 29 \\
\hline & São Francisco do Aiucá & 184 & 32 \\
\hline & São Paulo do Coraci & 75 & 14 \\
\hline & Sítio Fortaleza São José & 111 & 16 \\
\hline & Várzea Alegre & 82 & 13 \\
\hline & Vila Alencar & 131 & 25 \\
\hline & Vila Betel & 38 & 5 \\
\hline & Vila Nova do Amanã & 69 & 12 \\
\hline & Vila Nova do Coraci & 30 & 8 \\
\hline
\end{tabular}

Localidades sem Sistema de Abastecimento de Água (SAA) e que foi realizado exame parasitológico

- Localidades onde foi realizado exame parasitológico

Fonte: Instituto de Desenvolvimento Sustentável Mamirauá (IDSM). 
com uma "casinha" de madeira, construídas próximas ao domicílio. Outra forma de disposição usada é denominada regionalmente como "pau-da-gata", termo que significa defecar ao ar livre, geralmente adentrando a mata nas proximidades do domicílio para ter alguma privacidade.

No período da cheia a água inunda esses espaços, disseminando patógenos relacionados às doenças diarreicas, principalmente helmintos e protozoários. Quando o nível da água começa a diminuir (vazante - normalmente em meados de agosto), o solo contaminado com esses patógenos fica exposto e configura uma potente fonte de contaminação, que representa risco à saúde pela possibilidade da continuidade do ciclo parasito-hospedeiro $20,21$.

\section{Metodologia}

A coleta de dados foi realizada entre agosto de 2016 e fevereiro de 2017 em 21 comunidades inseridas na área de estudo, divididas em dois grupos de análise:

- Grupo 1: Análise de impactos em comunidades que receberam o SAA entre 2001 e 2014 ( $\mathrm{n}=19)$, incluindo: (a) 5 comunidades com SAA ativo: SAA em uso no momento de realização da pesquisa; e (b) 14 comunidades com SAA inativo: nestas o SAA parou de funcionar entre 2007 e 2014 e não foi reabilitado por diferentes motivos.

- Grupo 2: Análise da qualidade da água e da prevalência de parasitoses $(\mathrm{n}=6)$, incluindo: (c) 3 comunidades que não receberam o SAA e utilizam água diretamente do rio, para formar um subgrupo controle; $\mathrm{e}$ (d) 3 comunidades com SAA ativo, com características similares às comunidades do subgrupo controle, tais como corpo hídrico mais usado, número de domicílios e atividade econômica.

Os recursos metodológicos utilizados para avaliação dos impactos e o número de sujeitos participantes da pesquisa foram:

(I) Grupos focais 22,23 realizados entre abril e agosto de 2016, com a participação de 150 moradores das comunidades do Grupo 1, sendo um grupo focal por comunidade $(\mathrm{n}=19)$. A pergunta-chave da discussão foi: "Após a instalação do SAA, quais foram as principais mudanças que ocorreram na sua comunidade?", a fim de captar a percepção local sobre as mudanças e identificar impactos não previstos. Esses grupos focais foram gravados em áudio com a autorização dos participantes.

(II) Entrevistas semiestruturadas 22,23,24, realizadas entre novembro de 2016 e março de 2017, com um morador de cada comunidade do Grupo 1, sendo entrevistado um morador antigo de cada comunidade $(\mathrm{n}=19$ entrevistas). Teve como objetivo compreender as dificuldades cotidianas da comunidade. (III) Questionários 22,23 aplicados entre novembro de 2016 e março de 2017, de maneira censitária nos domicílios das comunidades do Grupo 1 ( $\mathrm{n}=217$ questionários) contendo 70 perguntas, agrupadas em (a) características do domicílio, (b) informações sobre saneamento, (c) hábitos diários e (d) ocorrência de doenças e acidentes.

(IV) Exame parasitológico realizado entre setembro e dezembro de $2016 \mathrm{em}$ moradores das comunidades do Grupo $2(\mathrm{n}=393)$ para o cálculo da prevalência de parasitas na população. As coletas de material para o exame foram realizadas durante a seca dos rios (meados de outubro até dezembro de 2016), período em que os moradores relataram perceber mais casos de doenças diarreicas ao longo dos anos. Os exames parasitológicos foram realizados em todos os moradores que concordaram com a pesquisa. As amostras de fezes foram analisadas em um laboratório de análises clínicas na cidade de Tefé, no Amazonas, que tem infraestrutura necessária para a realização da técnica do TF Test (BioBrasil; https://bio-brasil.com/tf-test/), e assim que concluídos os resultados foram sistematizados. E, submetidos ao teste de qui-quadrado e razão de chance (OR, com intervalo de $95 \%$ de confiança IC95\%; usando Microsoft Excel - https://products.office.com/), buscando identificar a influência do SAA na prevalência de monoparasitismo (helmintos ou protozoários) e poliparasitismo na população, com base em tabelas de contingência. Esses métodos foram usados em estudos similares, na análise de intervenções em qualidade da água 25 .

(V) Análise da qualidade da água por meio de amostras nas comunidades do Grupo 2 ( $\mathrm{n}=33$ amostras). As coletas foram realizadas duas vezes em cada comunidade. Entre setembro e dezembro de 2016 foram coletadas amostras de: água de beber, água de cozinhar, água do rio em frente à comunidade e, nas comunidades com SAA, também água da torneira. Os parâmetros analisados foram $\mathrm{pH}$ (pHmetro HQ40D, Hach; https://www.hexis.com.br/produto/medidor-multiparametro-digital- 
duplo-canal-hq4-1), turbidez (turbidímetro HI93703C, Hanna; https://hannainst.com.br/produto/ medidor-turbidez-com-maleta-para-transporte/), cloro livre (fotômetro HI97711C, Hanna; https:// hannainst.com.br/produto/hi97711c/), coliformes totais e Escherichia coli (filtração em membranas), considerados básicos na amostragem do padrão de qualidade da água do Ministério da Saúde 1. As análises seguiram os protocolos da Associação Americana de Saúde Pública (APHA) 26. Para identificar diferenças significativas na qualidade da água usada nas comunidades com e sem SAA foi realizado o teste U de Mann-Whitney, usando-se o software Statistica 10 (http://www.statsoft.com), com IC95\%.

\section{Questões éticas}

Este projeto foi avaliado pelo Comitê de Ética em Pesquisa com Seres Humanos do Instituto de Desenvolvimento Sustentável Mamirauá (CEP-IDSM), seguindo recomendações da Resoluções no 466/2012 e da Resolução no 510/2016 do Conselho Nacional de Saúde, sob o parecer no 2.442.553. O Termo de Consentimento Livre e Esclarecido foi lido para cada participante da pesquisa antes de ser assinado.

\section{Resultados e discussão}

As principais espécies parasitárias encontradas durante a pesquisa foram a Ascaris lumbricoides (77\% nas comunidades com SAA e $86 \%$ nas sem SAA) e Trichuris trichiura (28\% e 33\%), denominadas infecções helmínticas. Já entre os protozoários prevaleceram Giardia lamblia (27\% e 25\%) e Entamoeba histolytica (22\% e $30 \%)$.

$\mathrm{Na}$ Tabela 1 são apresentados os resultados de prevalência de helmintos e protozoários na população total. Os cálculos foram feitos com 393 exames parasitológicos, com base nos quais identificaram-se $99 \%$ e $100 \%$ de prevalência de pelo menos um parasito nas comunidades com e sem SAA, respectivamente, sendo mais frequente a presença de helmintos. São valores altos, principalmente quando comparados com outras regiões do Amazonas, tais como: 69\% de prevalência de parasitos no Rio Negro, em 1994 27; 76\% em áreas indígenas de São Gabriel da Cachoeira, em 2005 20; 44\% na região periférica de Manaus, em 2007 28; 66\% em Juruá, em 2007 29; e 83\% em Coari, em 200830.

No presente estudo, observa-se que a presença do SAA não levou à redução da prevalência total de parasitismo na população estudada. Por outro lado, baseando-se no teste qui-quadrado foi possível identificar na população com SAA menor chance de ocorrência de poliparasitismo (OR = 0,5; IC95\%: 0,3-0,8) e menor chance da ocorrência ( $\mathrm{OR}=0,5$; IC95\%: 0,3-0,7) de dois tipos de parasitas concomitantemente (protozoários + helmintos). A presença do SAA também resultou em menor chance de ocorrência de parasitismo por helmintos ( $\mathrm{OR}=0,3$; IC95\%: 0,1-0,7), porém, não apresentou influência significativa na presença de protozoários na população estudada.

A redução do poliparasitismo é um resultado positivo relevante. Apesar do efeito da presença de múltiplos parasitas ainda ser investigado, é possível que ocorra redução da resposta imunológica aos parasitas individualmente, aumentando a suscetibilidade clínica às doenças 31 . Sabe-se que o efeito do poliparasitismo é variável de acordo com a idade do indivíduo e a intensidade da infecção, podendo ter impactos aditivos ou multiplicativos de desnutrição, especialmente em crianças, levando, a longo prazo, a deficiências de crescimento e desenvolvimento cognitivo irreversíveis e até mesmo à morte 32 .

A transmissão desses parasitos ocorre de forma feco-oral, que abarca contato pelas mãos, alimentos, pés descalços, vetores e água. Dessa forma, todos os componentes do saneamento estão associados, incluindo o abastecimento de água. Mas a presença de saneamento não é suficiente ${ }^{33}$ para eliminar os riscos de adoecimento, uma vez que a transmissão de doenças ocorre nos domínios doméstico e público, o que demanda ações complementares de infraestrutura e educação em saúde, por exemplo.

Os condicionantes das infecções por protozoários foram estudados em diversos contextos ${ }^{34}$, identificando maiores chances de contaminação por G. lamblia em crianças consumindo água de poço e nascente em regiões urbanas pobres. Apesar do uso de água de cisternas reduzir a chance da ocorrência de G. lamblia em comunidades do semiárido brasileiro 35, outros fatores socioeconômicos estiveram relacionados, como o número de cômodos do domicílio, renda familiar, higiene das mãos e higiene dos alimentos com água não tratada. A disposição inadequada de esgoto e a construção de domicílios com madeira foram os fatores de maior influência para as protozooses em uma periferia urbana 28. 
Tabela 1

Prevalência de helmintos e protozoários na população total.

\begin{tabular}{|c|c|c|c|c|c|c|}
\hline \multirow[t]{2}{*}{ Infecção parasitária } & \multicolumn{2}{|c|}{$\begin{array}{l}\text { Com } \\
\text { abastecimento }\end{array}$} & \multicolumn{2}{|c|}{$\begin{array}{c}\text { Sem } \\
\text { abastecimento }\end{array}$} & \multirow{2}{*}{$\begin{array}{c}\text { Resultado de } \chi^{2} \\
\left(\chi^{2} \text { crítico: } 3,84\right) \text { com } \\
95 \% \text { de confiança }\end{array}$} & \multirow[t]{2}{*}{ OR (IC95\%) } \\
\hline & $\mathbf{n}$ & $\%$ & $\mathbf{n}$ & $\%$ & & \\
\hline Monoparasitismo & 111 & 44 & 40 & 29 & Significativo * & $1,9(1,2-3,0)$ * \\
\hline Helmintos & 89 & 80 & 36 & 90 & - & - \\
\hline Protozoários & 22 & 20 & 4 & 10 & - & - \\
\hline Poliparasitismo & 141 & 55 & 98 & 71 & Significativo ** & $0,5(0,3-0,8) * *$ \\
\hline Helmintos + helmintos & 48 & 34 & 27 & 28 & - & - \\
\hline Helmintos + protozoários & 81 & 57 & 69 & 71 & Significativo $* \star \star$ & $0,5(0,3-0,7) * * *$ \\
\hline Protozoários + protozoários & 12 & 9 & 2 & 2 & - & - \\
\hline Parasitismo por protozoários & 115 & 45 & 75 & 54 & Não significativo \# & - \\
\hline Parasitismo por helmintos & 218 & 85 & 132 & 96 & Significativo \#\# & $0,3(0,1-0,7) \# \#$ \\
\hline Negativo (nenhum parasita) & 3 & 1 & 0 & 0 & - & - \\
\hline Total & 255 & 100 & 138 & 100 & - & - \\
\hline
\end{tabular}

IC95\%: intervalo de 95\% de confiança; OR: razão de chances.

Fonte: elaboração própria a partir de dados da pesquisa.

Nota: $\mathrm{N}=393$ exames parasitológicos.

* Categorias de comparação: poliparasitismo + ausência de parasitismo;

** Categorias de comparação: monoparasitismo + ausência de parasitismo;

*** Categorias de comparação: somente helmintos + somente protozoários;

\# Categorias de comparação: somente helmintos + ausência de parasitismo;

\#\# Categorias de comparação: somente protozoários + ausência de parasitismo.

Por outro lado, os helmintos (ou geo-helmintos) têm parte de seu ciclo de vida no solo e por este motivo sua transmissão depende de contato com o solo contaminado 36 . Estudo 14 apontou que a prevalência e a intensidade do helminto A. lumbricoides tiveram relação com a disponibilidade de água e com o nível de serviço oferecido; os melhores resultados decorreram da existência de canalização interna de água e vaso sanitário com descarga, os piores ocorreram quando a presença de água no domicílio esteve associada ao uso de latrinas para a disposição de excretas.

Esses estudos contribuem para a compreensão dos resultados da prevalência de helmintos nas comunidades com SAA, onde a existência de água encanada acaba por reduzir a quantidade de deslocamentos até o rio e, portanto, o contato com o solo em algumas atividades de rotina, como o banho e a lavagem de utensílios domésticos. No entanto, em diversas outras atividades diárias todos estão susceptíveis ao contato com o solo potencialmente contaminado com ovos de helmintos.

Nas comunidades com SAA, 67\% das amostras de água de beber e 33\% das amostras de água de cozinhar eram provenientes da chuva. Nas comunidades sem SAA, 83\% da água de beber e 33\% da água de cozinhar eram provenientes da chuva. Nos demais casos, as águas usadas eram do rio (coletadas diretamente ou recebidas do SAA pela torneira). Na Figura 3, são apresentados os resultados da qualidade da água.

De forma geral, nas comunidades com SAA a qualidade da água da torneira apresentou resultados medianos melhores do que a água do rio, que é o local de captação do SAA, principalmente nos parâmetros turbidez (13uT na torneira e $37 \mathrm{uT}$ no rio, $65 \%$ de redução) e cor aparente (38uC na torneira e $98 \mathrm{uC}$ no rio, $61 \%$ de redução), resultado do tratamento preliminar componente do sistema. Os parâmetros E. coli e coliformes termotolerantes apresentaram diferença menor que 1 log entre as concentrações medianas da água do rio e da torneira, com concentrações entre 2,8 e 4,9UFC/100mL na água de torneira. Todos esses parâmetros indicam que o SAA não teve a capacidade de fornecer água potável, considerando os valores máximos permitidos no Brasil para a água de consumo humano 1 : ausência de E. coli, turbidez até 0,5 uT e cor até $15 \mathrm{uT}$. Na forma atual, a ingestão da água diretamente 
Figura 3

Resultados de qualidade da água e teste $U$ de Mann-Whitney.

3a) Escherichia coli

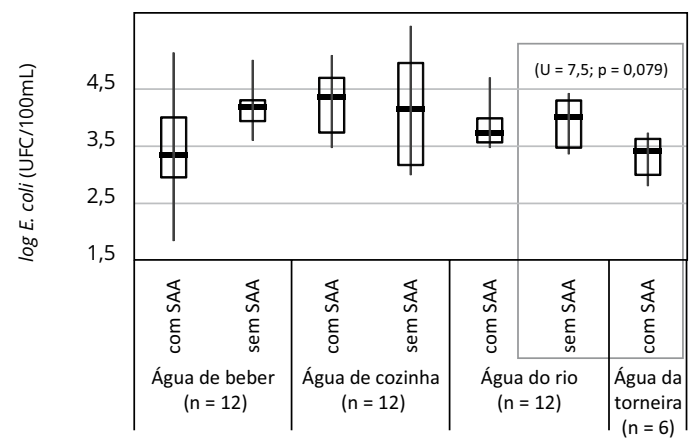

3c) Coliformes termotolerantes

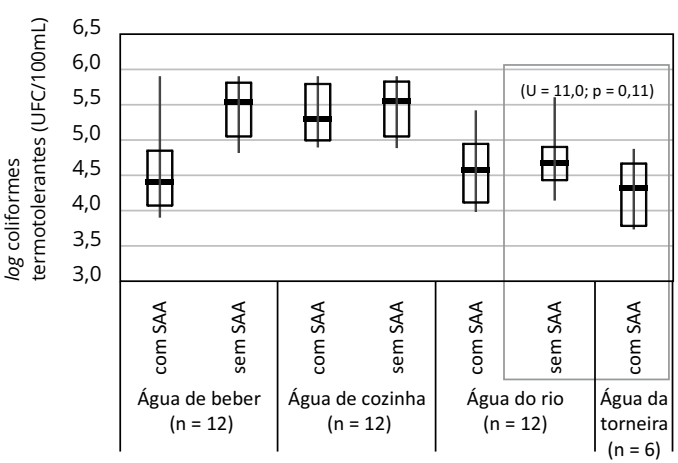

3b) Turbidez

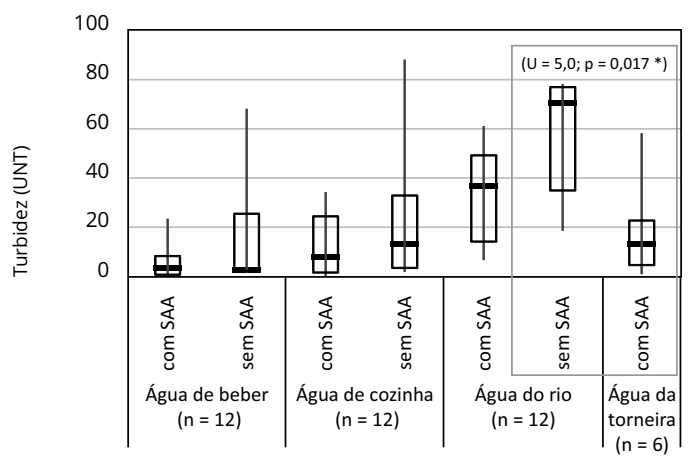

3d) Cor aparente

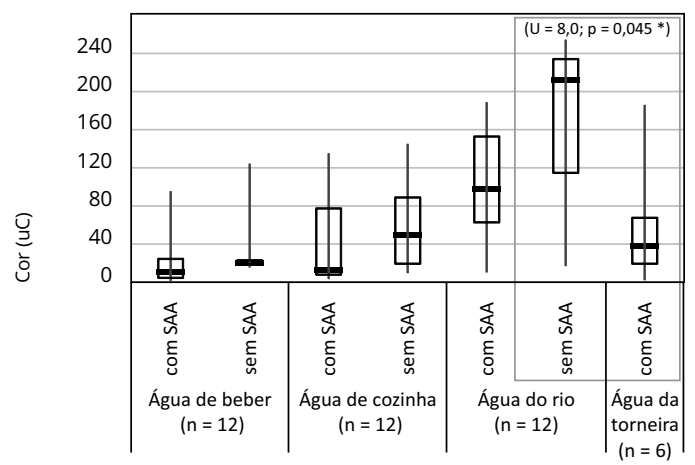

Fonte: elaboração própria a partir de dados da pesquisa.

* Diferenças significativas.

do SAA representa risco à saúde pela presença de E. coli e possivelmente outros microrganismos patogênicos, como protozoários e helmintos.

Na comparação entre a água do SAA (torneira) e a água do rio nas comunidades sem SAA, o teste de Mann-Whitney indicou haver diferença estatística significativa $(\mathrm{p}<0,05)$, com IC95\% para os parâmetros turbidez e cor aparente. As medianas indicam que a água nas comunidades com SAA tem melhor qualidade.

Segundo normas brasileiras 1 e recomendação da Organização Mundial da Saúde (OMS) 37, as águas superficiais necessitam ser no mínimo filtradas e desinfetadas antes do consumo. Uma vez que o SAA tem apenas filtração rudimentar, existe a necessidade de inclusão de outras etapas de tratamento, seja na rede de abastecimento, seja no domicílio (também chamado tratamento no ponto-de-uso), conforme previsto pela OMS em contextos específicos 37.

Outro impacto foi a diminuição do risco de acidentes relativos à ida até a beira do rio. No trajeto entre o domicílio e o rio, $44 \%$ dos entrevistados relataram acidentes ao descer os barrancos ou mesmo afogamento (principalmente de crianças) durante a rotina dos afazeres domésticos, além dos relatos sobre a diminuição do risco dos ataques de animais, pois 33\% dos entrevistados informaram que já foram atacados por algum deles (piranha, mandi, arraia, jacaré, cobra) realizando atividades fora do seu domicílio. 
Os impactos sociais dos SAAs foram relacionados às mudanças que ocorreram na rotina da família no consumo diário de água e aos investimentos realizados para a melhoria da infraestrutura do domicílio visando ao bem-estar do grupo doméstico. Esses impactos foram aferidos por meio de 11 indicadores e os resultados comparativos foram apresentados para as comunidades com SAA ativos e com SAA inativos, conforme exposto na Tabela 2.

Nas comunidades com SAA funcionando, as principais mudanças estão relacionadas à dinâmica de trabalho, à limpeza da casa e à higiene pessoal, conforme demonstrado na Tabela 2. Em depoimento, os moradores relataram sentirem-se mais à vontade para tomar banho dentro de casa, pela privacidade (como a possibilidade de ficar sem roupa) e por não terem o incômodo dos insetos, além do medo de ataque de animais na beira do rio, como relatado nos depoimentos a seguir.

"A gente às vezes ia tomar banho na beira e achava que ia voltar de lá frio. Mas voltava era suado e tinha carapanã [pernilongo, mosquito]. Agora com isso aí [SAA] melhorou muito" (Morador da comunidade Várzea Alegre, entrevista, 11/Abr/2016).

"Banho em terra é bom porque a gente pode tomar banho livre, só de calcinha, pode se lavar bem, e na beira não, lá tem que tomar banho com roupa e não pode tomar banho bem. Em casa a gente toma banho livre, às vezes de noite também. Não tem que descer pra beira, pode ter cobra pra morder a gente, e dentro de casa não" (Moradora da comunidade Ebenezer, entrevista, 17/Abr/2016).

Estudo realizado no Quênia, Tanzânia e Uganda 38 sugere que o menor volume de água coletado fora de casa apresenta um impacto negativo que aumenta os riscos de doenças infecciosas, como a diminuição da frequência de banho com higienização adequada ${ }^{39}$, a lavagem de roupas e vasilhas. Compromete ainda o tempo dedicado para outras atividades consideradas importantes, como o cuidado com os filhos, aprendizado e lazer 40

Nas comunidades com SAA ativo, 55\% dos entrevistados informaram que as mulheres começaram a realizar outras atividades após a instalação do SAA, como: produção de hortaliças, artesanato e lazer. Ou mesmo acordar mais tarde ou descansar por mais tempo entre uma atividade e outra, já que a água facilita a realização das tarefas domésticas.

"Agora com o sistema ajudou, facilitou para as mães que têm mais de um filho. Cuidar do almoço mais dar banho nos filhos atrasava o dia a dia em casa. Com tudo em casa, principalmente a água, se torna mais fácil

Tabela 2

Indicadores de mudança de bem-estar e de infraestrutura domiciliar.

\begin{tabular}{|c|c|c|c|}
\hline \multirow[t]{2}{*}{ Impactos } & \multirow[t]{2}{*}{ Indicadores } & \multicolumn{2}{|c|}{ Resultados (\%) } \\
\hline & & $\begin{array}{c}\text { SAA ativo } \\
(\mathrm{n}=60 \text { domicílios })\end{array}$ & $\begin{array}{c}\text { SAA inativo } \\
(\mathrm{n}=157 \text { domicílios })\end{array}$ \\
\hline Rotina e hábito de & Tomar banho em casa & 96,0 & 28,0 \\
\hline \multirow[t]{4}{*}{ consumo de água } & Lavar roupa e vasilhas em casa & 90,0 & 20,0 \\
\hline & Novas atividades realizadas pelas mulheres & 55,0 & - \\
\hline & Número de banhos diários (até 3 banhos ao dia) & 48,0 & 28,0 \\
\hline & Pegar água na beira do rio & 0,0 & 82,0 \\
\hline \multirow[t]{6}{*}{ Infraestrutura doméstica * } & Casas com caixa d'água & \multicolumn{2}{|c|}{27,2} \\
\hline & Casas com lavadora de roupa & \multicolumn{2}{|c|}{24,2} \\
\hline & Casas com banheiro & \multicolumn{2}{|c|}{12,9} \\
\hline & Casas com sanitários & \multicolumn{2}{|c|}{12,4} \\
\hline & Casas com pia & \multicolumn{2}{|c|}{11,1} \\
\hline & Novas ligações de água com torneira & \multicolumn{2}{|c|}{10,6} \\
\hline
\end{tabular}

SAA: Sistema de Abastecimento de Água.

Fonte: elaboração própria a partir de dados da pesquisa.

Nota: $\mathrm{N}=217$ questionários.

* Os dados de melhoria na infraestrutura representam esta melhoria na moradia após a instalação do SAA, independentemente do SAA estar ativo ou não no momento da avaliação. 
dar banho nos meninos e fazer comida, aprontava duas coisas de uma vez só". (Morador da comunidade Ebenezer, entrevista, 17/Abr/2016).

Dessa forma, o SAA provoca mudanças relacionadas ao tempo gasto nas atividades diárias, principalmente das mulheres, cujo tempo despendido na obtenção de água pode representar 15\% do tempo produtivo diário 15 .

Além disso, o transporte de água em recipientes na cabeça normalmente começa na infância, quando a ossatura não está totalmente formada 40. Dos 217 entrevistados (SAA ativo e inativo), 11\% informaram que um dos impactos mais significativos do sistema era não precisar carregar peso (água, vasilhas e roupas) da beira do rio até os domicílios, ou mesmo a diminuição de ocorrência de dores nas costas dos responsáveis pela coleta da água. Na literatura técnica, o relato de dores nas costas associado à falta de abastecimento de água é incerto 41,42 . Porém, a realização dessa atividade pode acarretar danos à saúde em longo prazo, como registrado no semiárido baiano, região na qual verificou-se que 96\% das mulheres e crianças entrevistadas relataram dores nas costas 43 .

O local no qual são executados os trabalhos domésticos também foi indicado como fator desencadeador de dores nas costas. Em razão da precária infraestrutura desses locais, os moradores permanecem grande parte do dia em postura inadequada (de cócoras) em cima de balsas de madeira. Nas comunidades com SAA ativo, há um predomínio das atividades realizadas em terra firme (próximo ao domicílio), em detrimento das outras formas. Já nas comunidades com SAA inativo, as atividades são realizadas predominantemente na beira do rio, nas balsas flutuantes, geralmente sem cobertura e expostas ao sol.

A melhoria na infraestrutura doméstica pode ser percebida principalmente nos investimentos realizados pelas famílias após a instalação do SAA. O principal item declarado foram as caixas d'água, adquiridas por $27,2 \%$ dos entrevistados. Esse investimento é especialmente importante porque promove a ampliação da capacidade de armazenamento de água pelas famílias e por proporcionar um armazenamento adequado. Entretanto, durante a pesquisa foram identificados locais impróprios de armazenamento e sem nenhum tipo de proteção, ou em locais com condições precárias de saneamento.

A aquisição de lavadoras de roupa representou o segundo investimento mais frequente, realizado por $24,2 \%$ dos entrevistados. As famílias relataram que a água encanada facilitou o uso do equipamento no trabalho doméstico, já que era possível fazer mais de uma atividade ao mesmo tempo. Entretanto, a aquisição de bens como máquina de lavar é influenciada tanto pelo acesso à água no domicílio como pelo poder aquisitivo da família.

Ainda foram relatados investimentos na construção de um local para tomar banho $(12,9 \%)$, outro para fazer as necessidades fisiológicas $(12,4 \%)$ e a instalação de pias de plástico $(11,1 \%)$ nos domicílios após a instalação dos SAA. A disponibilidade de água no domicílio torna possível a adoção desses itens simples, mas fundamentais para a mudança de hábitos de higiene diária, evidenciando a busca das famílias pelo conforto e bem-estar.

No entanto, ter a água em casa não diminuiu os riscos à saúde devido a disposição inadequada de dejetos humanos. Nas comunidades com o SAA inativo, 36\% despejam seus dejetos em fossas rudimentares e $41 \%$ diretamente no solo exposto ("pau-da-gata") atraindo vetores que podem transmitir doenças. Já nas comunidades com o SAA ativo, as fossas rudimentares representam 37\% e o "pau-da-gata" 23\%. Pesquisa realizada em uma comunidade indígena no Distrito de Iauaretê, no Amazonas 19,20, mostrou que os riscos de infecção aos quais a comunidade estava exposta eram o aumento populacional, a falta de saneamento básico e a manutenção de alguns hábitos sanitários tradicionais, como a disposição dos dejetos humanos e de resíduos em áreas peridomiciliares.

\section{Considerações sobre os impactos não previstos}

Os resultados da avaliação dos impactos da tecnologia de acesso à água indicaram que somente o abastecimento domiciliar não foi suficiente para trazer os benefícios esperados no momento da concepção desta intervenção, ou seja, a diminuição de parasitoses. Entretanto, deve-se levar em consideração que o abastecimento de água é um dos fatores responsáveis pela diminuição da proliferação de doenças de veiculação hídrica. 
Nas análises, deve-se considerar também os determinantes sociais e econômicos, hábitos culturais, particularidades locais, a rotina diária dos moradores e o interesse de adesão da população, fatores que apresentam grande relevância no controle de doenças relacionadas à água. Mesmo não ocorrendo a diminuição significativa das parasitoses, os SAAs possibilitaram uma melhoria em relação aos acidentes, quedas e ataques de animais que ocorriam no percurso dos moradores até a margem do rio para realizar tarefas diárias, como buscar água, tomar banho, lavar roupa, entre outras, como foi relatado tanto nos grupos focais quanto nas entrevistas realizadas.

A importância que os SAAs adquiriram no dia a dia das famílias usuárias ficou mais evidente nos impactos sociais relacionados ao conforto das tarefas domésticas e privacidade para a higiene pessoal. O SAA representa uma melhoria nas condições de vida dessa população quando proporciona uma economia no tempo desprendido para a obtenção de água ou em outras atividades domésticas como, por exemplo, lavar roupa, vasilhas e dar banho nas crianças, trabalhos estes realizados na margem do rio quando não há acesso à água no domicílio. Essa otimização do tempo gera benefícios relacionados ao trabalho, uma vez que o tempo disponível pode ser investido em outras atividades domésticas ou econômicas, como a produção de hortaliças ou até mesmo no lazer e conforto pessoal.

Ressalta-se ainda que os processos de avaliação de intervenções devem considerar fatores como o modo de vida da população usuária e outros aspectos socioeconômicos, que podem estar diretamente relacionados ao sucesso ou não de uma intervenção. Sistemas como esse configuram uma possibilidade para o início de uma solução para contextos rurais alagados, como o das comunidades ribeirinhas da Amazônia que convivem com as adversidades do ambiente, com as distâncias geográficas, com a baixa densidade demográfica e, principalmente, com a falta de políticas públicas adequadas para a região.

\section{Colaboradores}

A. C. N. Pacifico, I. M. Penteado e M. C. R. L. Gomes contribuíram com o desenvolvimento teórico-conceitual, a estruturação do artigo e a análise dos dados. A. C. S. Nascimento contribuiu com o desenvolvimento teórico-conceitual e a estruturação do artigo. D. S. S. Corrêa contribuiu com o desenvolvimento teórico-conceitual e a análise dos dados. J. P. B. Pedro e U. A. F. Gomes contribuíram com a análise e interpretação dos dados, a redação e a revisão crítica do conteúdo.

\section{Informações adicionais}

ORCID: Amanda Cristina Nunes Pacifico (00000002-2454-5377); Ana Claudeise Silva do Nascimento (0000-0002-3068-1324); Dávila Suellen Souza Corrêa (0000-0003-0442-5797); Iaci Menezes Penteado (0000-0002-1452-3836); João Paulo Borges Pedro (0000-0002-9735-8871); Maria Cecilia Rosinski Lima Gomes (0000-0003-0108-0148); Uende Aparecida Figueiredo Gomes (0000-00022750-8635).

\section{Agradecimentos}

Os autores agradecem ao Conselho Nacional de Pesquisa e Desenvolvimento (CNPq) pela concessão da bolsa do Programa de Capacitação Institucional (PCI) para a primeira e quarta autoras, ao Instituto de Desenvolvimento Sustentável Mamirauá (IDSM) pela infraestrutura de pesquisa, e a todas as famílias que nos receberam durante o período de coleta de dados, pela gentil acolhida e disponibilidade para participar da pesquisa. Financiadora de Estudos e Projetos (FINEP). 


\section{Referências}

1. Ministério da Saúde. Portaria de Consolidação no 5, de 28 de setembro de 2017. Consolidação das normas sobre as ações e os serviços de saúde do Sistema Único de Saúde. Diário Oficial da União 2017; 3 out.

2. Instituto Brasileiro de Geografia e Estatística. Censo Demográfico 2010: características da população e dos domicílios - resultados do universo. Rio de Janeiro: Instituto Brasileiro de Geografia e Estatística; 2011.

3. Gomes UAF, Heller L. Acesso à água proporcionado pelo Programa de Formação e Mobilização Social para Convivência com o Semiárido: um Milhão de Cisternas Rurais: combate à seca ou ruptura da vulnerabilidade? Eng Sanit Ambient 2016; 21:623-33.

4. Escola de Administração de Empresas de São Paulo, Fundação Getulio Vargas. Projeto Conexão local. Comunidades ribeirinhas e o projeto saúde e alegria no Estado do Pará. São Paulo: Escola de Administração de Empresas de São Paulo, Fundação Getulio Vargas; 2007.

5. Souza CMN, Nogueira AS, Vasconcelos ASS, Silva AS. Abastecimento de água em comunidades ribeirinhas da Amazônia brasileira e promoção da saúde: análise de modelo de intervenção e de gestão. Novos Cadernos NAEA 2012; 15:343-60.

6. Bernardes C, Bernardes RS. Caderno água, saneamento e saúde da população extrativista na Amazônia. Manaus: Memorial Chico Mendes; 2016.

7. Ramalho EE, Macedo J, Vieira TM, Valsecchi J, Calvimontes J, Marmontel M, et al. O ciclo hidrológico nos ambientes de várzea da Reserva de Desenvolvimento Sustentável Mamirauá Médio Rio Solimões, período de 1990 a 2008. UAKARI 2009; 5:61-87.

8. Wolf J, Prüss-Ustün A, Cumming O, Bartram J, Bonjour S, Cairncross S, et al. Assessing the impact of drinking water and sanitation on diarrhoeal disease in low- and middle-income settings: systematic review and meta-regression. Trop Med Int Health 2014; 19:928-42.

9. Gomes MCRL, Nascimento ACS, Corrêa DSS, Brito SO, Moura EAF. Surrounded by sun and water: development of a water supply system for riverine peoples in Amazonia. Revista Tecnologia e Sociedade 2019; 15:92-112.

10. Cohen E, Franco R. Avaliação de projetos sociais. 8a Ed. Petrópolis: Vozes Editora; 2008.

11. Sobsey MD, Stauber CE, Casanova LM, Brown JM, Elliott MA. Point of use household drinking water filtration: a practical, effective solution for providing sustained access to safe drinking water in the developing world. Environ Sci Technol 2008; 42:4261-7.

12. Tadesse A, Bosona T, Gebresenbet G. Rural water supply management and sustainability: the case of Adama Area, Ethiopia. J Water Resour Prot 2013; 5:208-21.
13. WaterAid. Looking back: the long-term impacts of water and sanitation projects. London: WaterAid; 2001.

14. Esrey SA. Effects of improved water supply and sanitation on ascariasis, diarrhoea, dracunculiasis, hookworm infection, schistosomiasis, and trachoma. Bull World Health Organ 1991; 59:609-21.

15. Heller 1, Pádua VL, organizadores. Abastecimento de água para consumo humano. 2a Ed. Belo Horizonte: Editora UFMG; 2010.

16. Andrade LC, Pereira HC, Nascimento ACS, Gomes MCRL. Os potenciais impactos da pandemia da COVID-19 nas comunidades ribeirinhas da Amazônia Central e as soluções recomendadas para mitigação. (Nota Técnica COVID-19 no 004, 30/04/2020). http://cloud. prodam.am.gov.br/index.php/s/U4hXTYiy GgmR6bn (acessado em 20/Mai/2020).

17. Moura EAF, Nascimento ACS, Corrêa DSS, Alencar EF, Sousa IS. Sociodemografia da Reserva de Desenvolvimento Sustentável Mamirauá: 2001-2011. http://livroaberto.ufpa. br/jspui/handle/prefix/14 (acessado em 19/ Fev/2019).

18. Moura EAF. Água de beber, água de cozinhar, água de tomar banho: diversidade socioambiental no consumo da água pelos moradores da várzea de Mamirauá, Estado do Amazonas. Cad Saúde Colet (Rio J.) 2007; 15:501-16.

19. Giatti LL, Rocha AA, Toledo RF, Barreira LP, Rios L, Pelicioni MCF, et al. Condições sanitárias e socioambientais em Iauaretê, área indígena em São Gabriel da Cachoeira - AM. Ciênc Saúde Colet 2007; 12:1711-23.

20. Rios L, Cutolo SA, Giatti LL, Castro M, Rocha AA, Toledo RF, et al. Prevalência de parasitos intestinais e aspectos socioambientais em comunidade indígena no Distrito de Iauaretê, Município de São Gabriel da Cachoeira (AM), Brasil. Saúde Soc 2007; 16:76-86.

21. Cutolo SA, Matté MH, Rocha AA. Monitoring of parasitological contamination in treated wastewater from activated sludge system. Management of Environmental Quality 2006; 17:43-56.

22. Morgan D. Focus group as qualitative research. London: Sage Publications; 1997. (Qualitative Research Methods Series, 16).

23. Giatti BA. Grupo focal na pesquisa em Ciências Sociais e Humanas. Brasília: Líber Livro; 2005.

24. Drumond MA, Giovanetti L, Guimarães A. Técnicas e ferramentas participativas para a gestão de unidades de conservação. Brasília: Programa Áreas Protegidas da Amazônia/Ministério do Meio Ambiente; 2009. (Cadernos ARPA, 4).

25. Gundry S, Wright J, Conroy R. A systematic review of the health outcomes related to household water quality in developing countries. J Water Health 2004; 2:1-13. 
26. American Public Health Association. Standard methods for the examination of water and wastewater. 21st Ed. Washington DC: American Public Health Association/American Water Works Association/Water Environment Federation; 2005.

27. Coura JR, Willcox HPF, Tavares AM, Paiva DD, Fernandes O, Rada ELJC, et al. Aspectos epidemiológicos, sociais e sanitários de uma área no Rio Negro, Estado do Amazonas, com especial referência às parasitoses intestinais e à infecção chagásica. Cad Saúde Pública 1994; 10 Suppl 2:327-36.

28. Visser S, Giatti LL, Carvalho RAC, Guerreiro JCH. Estudo da associação entre fatores socioambientais e prevalência de parasitose intestinal em área periférica da Cidade de $\mathrm{Ma}$ naus - AM. Ciênc Saúde Colet 2011; 16:348192.

29. Bernardes C. Avaliação integrada de impacto à saúde decorrente de ações de saneamento em comunidades de unidades de conservação de uso sustentável na Amazônia [Tese de Doutorado]. São Paulo: Universidade de São Paulo; 2013.

30. Santos FS, Gama ASM, Fernandes AB, Reis Junior AB, Guimarães J. Prevalência de enteroparasitismo em crianças de comunidades ribeirinhas do Município de Coari, no médio Solimões, Amazonas, Brasil. Rev Pan-Amazônica Saúde 2010; 1:23-8.

31. Chammartin F, Scholte RGC, Guimarães LH, Tanner M, Utzinger J, Vounatsou P. Soil-transmitted helminth infection in South America: a systematic review and geostatistical metaanalysis. Lancet Infect Dis 2013; 13:507-18.

32. Pullan R, Brooker S. The health impact of polyparasitism in humans: are we under-estimating the burden of parasitic diseases? Parasitology 2008; 135:783-94.

33. Cairncross S, Blumenthal U, Kolsky P, Moraes L, Tayeh A. The public and domestic domains in the transmission of disease. Trop Med Int Health 1996; 1:27-34.

34. Teixeira JC, Heller L, Barreto ML. Giardia duodenalis infection: risk factors for children living in sub-standard settlements in Brazil. Cad Saúde Pública 2007; 23:1489-93.

35. Fonseca JE, Carneiro M, Pena JL, Colosimo EA, Silva NB, Costa AG, et al. Reducing occurrence of Giardia duodenalis in children living in semiarid regions: impact of a large scale rainwater harvesting initiative. PLoS Negl Trop Dis 2014; 8:e2943.
36. Bopda J, Nana-Djeunga H, Tenaguem J, Kamtchum-Tatuene J, Gounoue-Kamkumo R, Assob-Nguedia C, et al. Prevalence and intensity of human soil transmitted helminth infections in the Akonolinga health district (Centre Region, Cameroon): are adult hosts contributing in the persistence of the transmission? Parasite Epidemiol Control 2016; 1:199-204.

37. World Health Organization; United Nations Children Fund. Guidelines for drinking-water quality. http://www.who.int/water_sanita tion_health/publications/drinking-waterquality-guidelines-4-including-1st-adden dum/en/ (acessado em 01/Set/2018).

38. Thompson J, Porras IT, Tumwine JK, Mujwahuzi MR, Katui-Katua M, Johnstone N, et al. Drawers of water II: 30 years of change in domestic water use and environmental health in East Africa. London: International Institute for Environment and Development; 2001.

39. Howard G, Bartram J. Domestic water quantity, service level and health. Geneva: World Health Organization; 2003.

40. Razzolini MTP, Günther WMR. Impactos na saúde das deficiências de acesso à água. Saúde Soc 2008; 17:21-32.

41. Hunter PR, Resebro H. Defining the current situation - epidemiology. In: Cameron J, Hunter P, Jagals P, Pond K, editors. Valuing water, valuing livelihoods: guidance on social cost-benefit analysis of drinking-water interventions, with special reference to small water supplies. London: World Health Organization/ IWA Publishing; 2011. p. 75-100.

42. Pond K, Pedley S. Current situation in access to drinking-water. In: Cameron J, Hunter P, Jagals P, Pond K, editors. Valuing water, valuing livelihoods: guidance on social cost-benefit analysis of drinking-water interventions, with special reference to small water supplies. London: World Health Organization/IWA Publishing; 2011. p. 55-74.

43. Orrico SEM. Sistema associativo de saneamento e seus efeitos sobre a população em comunidades do semi-árido baiano [Tese de Doutorado]. São Paulo: Faculdade de Saúde Pública, Universidade de São Paulo; 2003. 


\section{Abstract}

The study presents an assessment of the health and social impacts from the implementation of water supply systems in riverine communities in the Middle Solimões region, Amazonas State, Brazil. Data were collected in 21 rural communities, 19 of which received the water access technology experimentally in the last 14 years. For comparison, three rural communities that had not received the technology participated in the study. A quantitative/qualitative methodology was used with the following techniques: focus groups, semi-structured interviews, questionnaires, parasitological tests, and water quality analysis. Even without a reduction in the prevalence of parasite infections, the systems showed impacts on comfort, privacy, and reduction in the time spent on household chores such as fetching water or having to wash clothes and pots and pans outdoors and far from home. The impacts point to an improvement in living conditions from the perspective of wellbeing.

Water Supply; Amazonian Ecosystem;

Sustainable Development; Water

\section{Resumen}

Este estudio presenta una evaluación de los impactos de salud y social, a partir de la implementación de sistemas de abastecimiento de agua en comunidades ribereñas en la región del Médio Solimões, estado de Amazonas, Brasil. Los datos se recabaron en 21 comunidades rurales, entre las cuales 19 contaron con tecnología de acceso al agua de forma experimental durante los últimos 14 años. Para la comparación, participaron en la investigación tres comunidades rurales que no recibieron esta tecnología. La metodología utilizada fue cuanti/cualitativa, con aplicación de las siguientes técnicas: grupos focales, entrevistas semiestructuradas, cuestionarios, exámenes parasitológicos, así como análisis de la calidad del agua. Se evaluó que, incluso no produciéndose la reducción de la prevalencia de parasitosis, los sistemas presentaron impactos en cuanto al confort, la privacidad $y$ en la reducción del tiempo empleado en actividades domésticas, como buscar agua en el río o lavar la ropa y la vajilla fuera del domicilio. Los impactos apuntan hacia una mejoría de las condiciones de vida, desde el punto de vista del bienestar.

Abastecimiento de Agua; Ecosistema Amazónico; Desarrollo Sostenible; Agua
Recebido em 27/Abr/2020

Versão final reapresentada em 17/Jul/2020

Aprovado em 31/Jul/2020 